
sector servicios en Colombia. Económicas CUC, 37(2), 89114. DOI: http://dx.doi.org/10.17981/econcuc.37.2.2016.05

\title{
Financiación de la innovación tecnológica en el sector servicios en Colombia ${ }^{1}$
}

DOI: http://dx.doi.org/10.17981/econcuc.37.2.2016.05

\section{Julio Cesar Caro Moreno²}

\section{Resumen}

En el contexto actual, donde la innovación se ha convertido en una variable importante a la hora de abordar temas relacionados con el crecimiento económico, surgió la necesidad de estudiar de forma detallada los procesos de innovación generados en el país, con el fin de identificar las variables que influyen directa e indirectamente en la determinación del proceso productivo. Para este artículo en particular, la financiación en la innovación tecnológica en el sector servicios en Colombia periodo 2008 - 2011. Se abarca el sector servicios, debido no solo a la tendencia mundial hacia la tercerización de la economía, sino también a su creciente participación en el Producto Interno Bruto (PIB). En la primera parte se plantean los aspectos más relevantes con respecto al tema de innovación. Posteriormente, se realiza un análisis del comportamiento del financiamiento de la innovación en el sector servicios, basados en los datos suministrados por el Departamento Administrativo Nacional de Estadística (DANE) a través de las Encuestas de Desarrollo e Innovación Tecnológica (EDITS).

Palabras clave: financiación; innovación tecnológica; sector servicios; Colombia.

Recibido: 29.9.2016 Devuelto para revisión: 7.11.2016 Aceptado: 29.11.2016

\footnotetext{
${ }^{1}$ Artículo científico derivado de la investigación "Innovación y cambio técnico en el sector industrial y servicios en Colombia. 2000-2010”, financiada por la Universidad Pedagógica y Tecnológica de Colombia.

${ }^{2}$ Doctorando en Geografía. Magister en Economía. Economista. Especialista en Finanzas. Universidad Pedagógica y Tecnológica de Colombia. Profesor investigador adscrito al grupo SOECOL de la Universidad Pedagógica y Tecnológica de Colombia. julio.caro@uptc.edu.co
}

- The author; licensee Universidad de la Costa - CUC. 


\title{
Funding of technological innovation in the services sector in Colombia
}

\begin{abstract}
In the current context, where innovation has become an important variable in addressing issues related to economic growth, the need arose to study in detail the innovation processes generated in the country, in order to identify the variables that directly and indirectly influence the determination of the productive process. For this article, financing in technological innovation in the services sector in Colombia period 2008 - 2011. The services sector is encompassed, due not only to the global trend towards outsourcing of the economy, but also to its growing participation in the Gross Domestic Product (GDP). First part, the most relevant aspects regarding the topic of innovation will be discussed. Subsequently, an analysis of the innovation financing behavior in the services sector is carried out based on data provided by the National Administrative Department of Statistics (DANE) through the Technological Development and Innovation Surveys (EDITS).
\end{abstract}

Keywords: funding; technological innovation; service sector; Colombia.

\section{Introducción}

El sector de servicios ha liderado el panorama económico en las últimas décadas y se ha convertido en la fuente principal de empleo, tanto en los países desarrollados como en aquellos en vías de desarrollo, por ejemplo, para el caso colombiano, el sector de servicios en 2010 representó el $65 \%$ del PIB interno y empleó en promedio el $67 \%$ del total de la población ocupada durante la última década.

De forma paralela, la innovación ha pasado a ser una de las variables de estudio más importantes en las últimas décadas, 
ya que es asociada con la generación del crecimiento, de conocimiento interno y de ventajas competitivas. Lo anterior ha llevado a la innovación a ser uno de los temas principales de la política pública mundial; tal como lo plantea Porter (1990, p. 73): “...la competitividad de una nación depende en la capacidad de su economía para innovar y mejorar."

Si bien se ha recopilado gran cantidad de datos e información de innovación por más de cinco décadas y se han hecho grandes esfuerzos en el contexto mundial para obtener indicadores de innovación desde lo micro y macro, aún no se tiene un amplio entendimiento de las colaboraciones y complejas interrelaciones entre la innovación y el sector de servicios.

En las últimas décadas, Colombia, al igual que la mayoría de los países latinoamericanos, ha venido generando diferentes encuestas de innovación en el sector manufacturero basadas en manuales internacionales y encaminadas a revelar las variables fundamentales y las interacciones de éstas dentro del proceso innovador.

El presente artículo hace un análisis de la Encuesta de Desarrollo e Innovación Tecnológica del sector servicios 20082011 con el fin de analizar el comporta- miento de la financiación por parte del sector privado, público y recursos propios de las empresas en Colombia.

Inicialmente se hace una revisión de los planteamientos encontrados en la literatura sobre los conceptos de servicios e innovación. Más adelante se presenta un análisis del comportamiento del PIB. Luego, se presenta el comportamiento del sector de servicios en Colombia junto con la evolución que ha tenido el financiamiento de la innovación por parte del sector de servicios. Por último, se presentan las conclusiones sobre los aportes de la financiación de la innovación en el sector de servicios en Colombia.

\section{Teoría económica e innovación}

Una de las grandes discusiones a las que se ha enfrentado la teoría económica a través de los años ha sido la determinación de las fuentes de crecimiento y desarrollo dentro de una economía. La mayoría de los estudios en la actualidad concuerdan en reconocer a la innovación como un factor fundamental para el crecimiento y desarrollo de un país, asimismo, identifican a la innovación como un factor primordial en la determinación de competitividad dentro del contexto del modelo de globalización. 
Las primeras contribuciones referentes a innovación son atribuidas a Schumpeter, quien fue uno de los primeros en identificar a la innovación como fuente de crecimiento dentro de una serie de conceptos evolucionistas donde el aumento del producto era vinculado estrechamente con las innovaciones que aparecían en la producción; a partir de esto, se vincularon otros factores al crecimiento económico, como la tecnología y la innovación originando nuevas ideas en las teorías de crecimiento.

Los teóricos de la innovación fundamentan su análisis en las aportaciones de Joseph Schumpeter, en las cuales el equilibrio no existe:

La economía capitalista no es ni puede ser estacionaria. Tampoco se expande conforme a un ritmo uniforme. Está, incesantemente, revolucionada desde dentro por un nuevo espíritu de empresa, es decir, por la introducción de nuevas mercancías o nuevos métodos de producción o nuevas posibilidades comerciales en la estructura industrial, tal como existe en cualquier momento. Todas las estructuras existentes y todas las condiciones de vida económica se hallan siempre en un proceso de transformación, toda situación es derribada antes de que haya tenido tiempo de desarrollarse plenamente. En la sociedad capitalista el progreso económico significa derrumbamiento (Schumpeter, 1971).
Por ende, las innovaciones tecnológicas irrumpen de manera explosiva en los procesos productivos concentrándose en algunos sectores, su distribución no es aleatoria, en consecuencia, son discontinuas, desequilibradas y de naturaleza no armónica, imprimiendo un carácter dinámico a la competencia empresarial. Su difusión es desigual entre los sectores y al interior de cada sector, debido al comportamiento cíclico en las diferentes fases: expansión, contracción, depresión y recuperación (Freeman, 1994).

La causa de las fluctuaciones económicas, para Schumpeter, es el proceso de innovación definido como un:

(...) proceso de mutación que revoluciona incesantemente la estructura económica desde dentro, destruyendo ininterrumpidamente lo antiguo y creando continuamente elementos nuevos. Este proceso de destrucción creadora constituye el elemento esencial del capitalismo. En ella consiste, en definitiva, el capitalismo y toda empresa capitalista tiene que amoldarse a ella para vivir (Schumpeter, 1971).

La competencia a través de la innovación tecnológica se determina por la capacidad que tiene cada economía para generar de manera continua nuevos productos y servicios que le aseguren una ganancia al em- 
presario. Esta competencia "imperfecta" entre las economías

fundamentalmente en términos de innovaciones industriales, constituye la base del desarrollo económico (...) el énfasis aparece en el lado de la oferta, es decir, en la inversión autónoma (...) se contempla el desarrollo económico fundamentalmente como un proceso de reasignación de los recursos entre las industrias. Este proceso provoca automáticamente cambios estructurales y desequilibrios, aunque sólo sea por la existencia de una distinta tasa de cambio tecnológico desigual en cada industria (Freeman, 1988).

Para Ruttan (1979), el concepto de innovación ha tenido mayor importancia a la que tiene la invención, además, considera que la primera alcanzó una mayor proyección a partir de que Schumpeter (1979) la identificó como la función esencial del empresario. Citando a éste señala que:

La innovación es posible sin nada que podamos identificar como invención, y la invención no induce necesariamente innovación, sino que en sí misma puede no producir ningún efecto económicamente importante en absoluto (Ruttan, 1979).

En la literatura se encuentra el planteamiento de dos momentos en el pensamiento schumpeteriano (Freeman, Clark y Soe- te, 1985). En el modelo I de Schumpeter ${ }^{1}$, es relevante el comportamiento arriesgado de las economías, las cuales, para innovar, se apoyaban en los adelantos de la ciencia y la tecnología exógenas a las empresas y a las estructuras de mercado que le proporcionan una renta monopólica, objetivo fundamental del empresario por un tiempo determinado, hasta que penetran empresas imitadoras como consecuencia de la difusión de las innovaciones. Cabe señalar que los que pueden imitar o copiar son aquellos empresarios que tienen condiciones o capacidades próximas a las del innovador. Al disminuir los beneficios se puede llegar a una etapa de estancamiento y depresión, la cual puede ser evitada por una nueva ola de innovaciones.

En el modelo II, considera a las actividades científicas y tecnológicas como un factor endógeno, realizadas por las grandes economías, como su principal estrategia competitiva llevando a cabo actividades de investigación y desarrollo (I+D). Al incrementarse estas actividades, aumenta la propensión a innovar, cuyos efectos son los siguientes: mayor concentración de mercado, nuevos

\footnotetext{
1 El primero es el que desarrolló antes de la Segunda Guerra Mundial, y lo presenta en su obra Theory of Economic Development (1912); el segundo modelo, lo expone en su libro, Capitalism, Socialism and Democracy (1943).
} 
modelos de producción, transformación de la estructura de mercado e incertidumbre por los resultados positivos o negativos (pérdidas o ganancias). Estas dos posiciones son complementarias, sin embargo, las innovaciones no surgen únicamente de las actividades de $\mathrm{I}+\mathrm{D}$, pueden ser originadas por las tareas rutinarias que van acumulando conocimiento, esto es, aprendizaje tecnológico.

\section{Una aproximación al concepto de innovación en el sector de servicios}

El tema del estudio de la innovación en el sector de servicios ha evolucionado a través del tiempo. Para el ISEA (2008), esta evolución se ha desarrollado en cuatro etapas: indiferencia, subordinación o asimilación, autonomía o demarcación y síntesis.

En la etapa de indiferencia, en la década de los ochenta, los servicios no se consideraban innovadores, por lo que no se encuentran estudios. Posteriormente, en los años noventa, el tema va adquiriendo relevancia y se aplican conceptos relacionados con el sector manufacturero para analizar la innovación en el sector de servicios, denominada la etapa de la subordinación o asimilación.
Después se efectúan cambios en la concepción de la innovación en servicios en la etapa de autonomía o demarcación, donde se considera que este sector es totalmente diferente al sector manufacturero, por lo cual se requieren nuevas bases teóricas adaptadas a sus características propias. Finalmente, llega la etapa de síntesis donde los estudios y la realidad demuestran que la innovación en servicios tiene más similitudes que discrepancias con la innovación en el sector manufacturero y en los estudios se empieza a relacionar esto.

\section{La innovación desde la perspectiva del Manual de Oslo}

El Manual de Oslo de la Organización para la Cooperación y el Desarrollo Económico (OCDE) recoge los conceptos, definiciones y metodología utilizados para la elaboración de datos sobre innovación tecnológica. Este manual inicialmente fue enfocado hacia las industrias manufactureras, por lo que los conceptos de innovación tecnológica se fundamentan en la innovación de procesos y productos con muy escaza referencia en innovaciones no tecnológicas o de otros sectores.

Según el Manual de Oslo (UNED, 2010), 
una innovación tecnológica comprende nuevos productos y procesos, así como cambios tecnológicos significativos de productos y procesos. Una innovación ha sido implementada si ha sido introducida en el mercado (innovación de producto) o utilizada dentro del proceso de producción (innovación de proceso). Las innovaciones por tanto implican una serie de actividades científicas, tecnológicas, organizativas, financieras y comerciales.

No obstante, la distinción entre productos y procesos en el sector de servicios es poco clara, ya que la producción y el consumo ocurren simultáneamente. Por esta razón, en su tercera edición, agregan la ampliación del concepto de producto donde se empieza a referir no sólo a bienes sino también a servicios:

La actividad de innovación en este sector (servicios) es un proceso continuo, consistente en una serie de modificaciones que se introducen progresivamente en los productos y en los procesos, con una fase inicial, la cual consta de investigación, evaluación de ideas y confrontación comercial, lo que lleva a una modificación importante en los productos, procesos o métodos" (UNED, 2010).

Así, se incorporan las siguientes definiciones de las diferentes clases de innovación:
Innovación de producto en el sector de servicios. Son servicios cuyo uso intencionado o características de estructura difieren significativamente de los servicios ya producidos. Las innovaciones serán el resultado de inversiones substanciales en nuevo conocimiento, sea o no tecnológico.

Innovación de proceso en el sector de servicios. Son formas nuevas o mejoradas de manera significativa de producir o proveer servicios. Por tanto, las innovaciones de proceso pueden ser innovaciones en los procesos de producción de los servicios o en los procesos de distribución o entrega (provisión).

Innovación organizativa en el sector de servicios. Se refiere a la puesta en práctica de nuevos métodos de organización, como cambios en las prácticas de la empresa, organización del lugar de trabajo o en las relaciones externas de la empresa. Además, dentro de este tipo de innovación se encuentra la innovación de mercadotecnia que implica la utilización de nuevos métodos de comercialización y nuevos comportamientos en el mercado, como por ejemplo un nuevo segmento de mercado o la introducción en otra industria. 
A pesar que en la teoría están descritas estas innovaciones, en el sector de servicios se dificulta su identificación particular debido a que la mayoría de las innovaciones presentan un poco de cada uno de estos tipos; además, el carácter no almacenable e intangible de los servicios ha contribuido al desinterés por el estudio del tema, desviando este interés hacia los sectores tradicionalmente productivos.

\section{Definición y clasificación del sector de servicios}

El sector de servicios o sector terciario hace parte de los tres grandes sectores en los que se encuentra dividida la economía, agrupando diversas actividades que lo caracterizan como el más heterogéneo dentro de las ramas productivas. Difiere del sector industrial y agropecuario en que su oferta radica en la prestación de servicios, los cuales son intangibles y consumidos en el instante en el cual son realizados, a diferencia de los bienes de los demás sectores los cuales son tangibles y almacenables; además, es el conector entre las actividades del sector primario $\mathrm{y}$ secundario.

Existen varias clasificaciones del sector de servicios debido a la heterogeneidad que éste presenta, por lo que en este caso nos basaremos en la clasificación utilizada por el DANE, el cual utiliza la Clasificación Industrial Internacional Unificada (CIIU) dada por la Organización de Naciones Unidas (ONU) para facilitar la recopilación y presentación de informes estadísticos. Esta clasificación (CIIU) ha sido adaptada por el DANE a las características de la economía colombiana con el objetivo de organizar de manera jerárquica y eficaz la información de las actividades económicas y, así, facilitar investigaciones y análisis que contribuyan a la elaboración de políticas públicas referentes al crecimiento de cada uno de los sectores de la economía.

\section{Estructura y evolución del sector de servicios en Colombia}

El sector de servicios se dedica a actividades económicas que no se relacionan directamente con el proceso de producción; están orientadas principalmente a la prestación de servicios necesarios para el funcionamiento de los demás sectores de la economía, como es el caso de los servicios financieros, de educación, transporte, comercio, telecomunicaciones y construcción, entre otros. En las últimas dos décadas, el sector de servicios aumentó su participación en el Producto Interno Bru- 
Tabla 1

Clasificación Industrial Internacional Unificada para Colombia (CIIU)

\begin{tabular}{cc}
\hline Clasificación CIIU & Descripción \\
\hline División 40 & Suministro de electricidad, gas, vapor y agua caliente \\
División 41 & Captación, depuración y distribución de agua \\
División 50 & Comercio de vehículos automotores, combustibles y lubricantes \\
División 51 & Comercio al por mayor, excepto el comercio de vehículos automotores \\
División 52 & Comercio al por menor, excepto el comercio de vehículos automotores \\
Grupos 551 y 552 & Hoteles y restaurantes \\
Grupos 602 y 604 & Transporte de pasajeros por vía terrestre y de carga por carretera \\
División 62 & Transporte por vía aérea \\
Grupos 641 y 642 & Correos y telecomunicaciones \\
Clase 6511 y 6512 & Actividades bancarias \\
División 72 & Informática \\
División 73 & Centros de investigación y desarrollo \\
Grupo 805 & Educación superior privada \\
Grupo 851 & Actividades relacionadas con la salud humana \\
División 90 & Eliminación de desperdicios y aguas residuales, saneamiento y similares \\
Grupo 921 & Activides de cinematografía, radio y televisión y otras. \\
\hline
\end{tabular}

Fuente: DANE- EDIT (2005, 2007, 2009, 2011, 2013).

to (PIB), pasando de un $63 \%$ en la década de los noventa a un $65 \%$ en la década del dos mil, generando el $67 \%$ del empleo en la economía colombiana, como lo revela el
DANE en la Gran Encuesta Integrada de Hogares (GEIH); por esta razón, las expectativa por parte del Gobierno se dirigieron hacia este sector. 


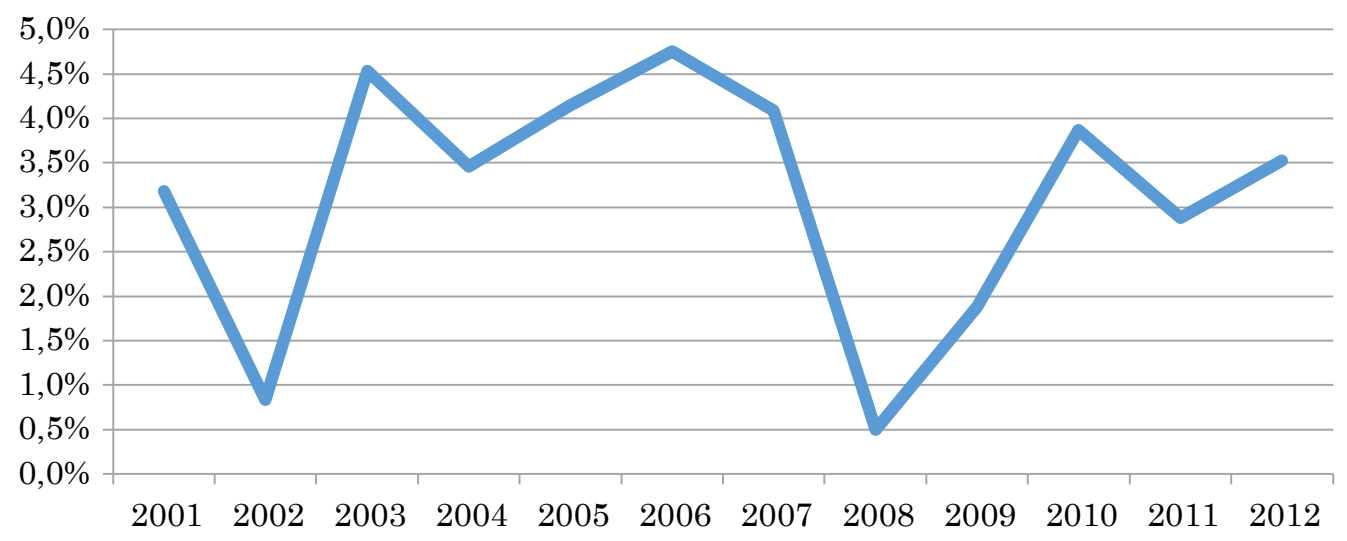

Gráfico 1. Crecimiento anual del sector de servicios para la década del 2000.

Fuente: elaboración propia a partir del DANE (2005, 2007, 2009, 2011, 2013).

El sector de servicios en la década del 2000 tuvo la mayor participación en el PIB con respecto a los demás sectores de la economía colombiana tradicionalmente productivos, su participación fue de un $64 \%$ en relación con los demás sectores, como se puede observar en el gráfico 1, donde el aporte del sector de servicios supera la suma del aporte de los demás sectores que componen la economía colombiana, convirtiéndose en el sector jalonador de la economía colombiana, siguiendo los parámetros de la economía mundial hacia la tercerización.

Examinando el crecimiento en la década, se observa cómo el sector de servicios, aun sin ser el que refleja el mayor crecimiento, es el sector que presenta un comportamien- to estable con respecto a los otros sectores, inclusive a pesar de los rezagos de la crisis económica mundial causada por la burbuja inmobiliaria en Estados Unidos y la crisis nacional causada por las captadoras ilegales de dinero (pirámides).

En 2008 y 2009 fue el sector con menos impacto con relación a los demás sectores que se vieron afectados; como lo muestra el gráfico 3, el sector de servicios pasó de un crecimiento del $3,46 \%$ al 2,09\% disminuyendo en un $1,37 \%$ en su peor momento, mientras los sectores más afectados fueron el de la construcción que pasó de un $5,28 \%$ a un $-0,10 \%$, registrando una caída de $5,38 \%$, $\mathrm{y}$, por su parte, la industria de $6,59 \%$ en 2008 y $4,76 \%$ en 2009 . 

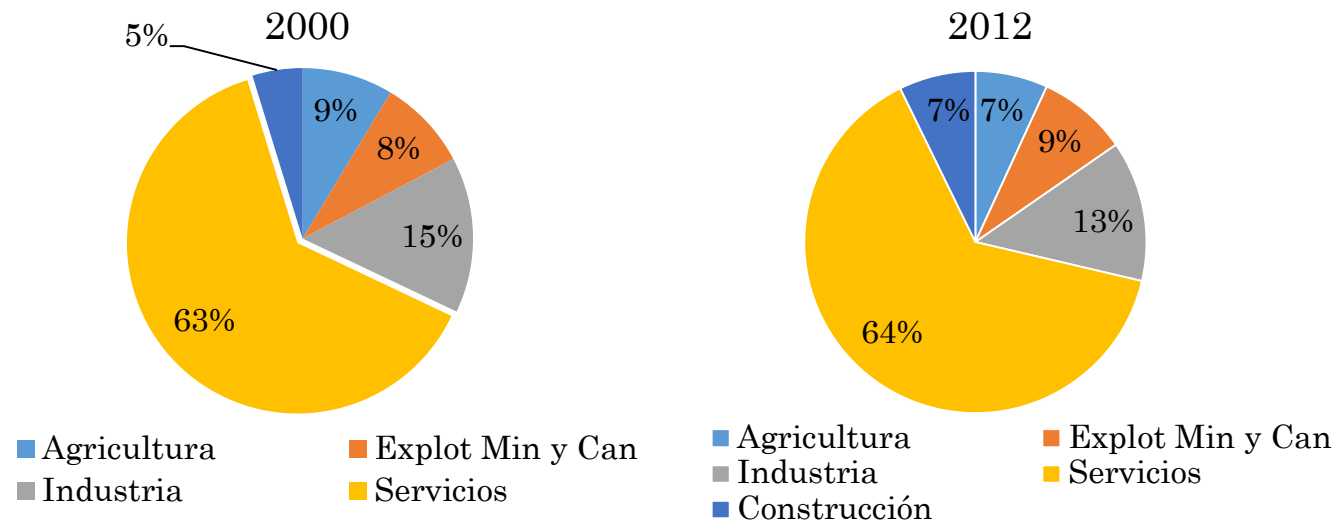

Gráfico 2. Participación por ramas de actividad económica en los años del 2000 y 2012.

Fuente: elaboración propia a partir del DANE (2005, 2007, 2009, 2011, 2013).

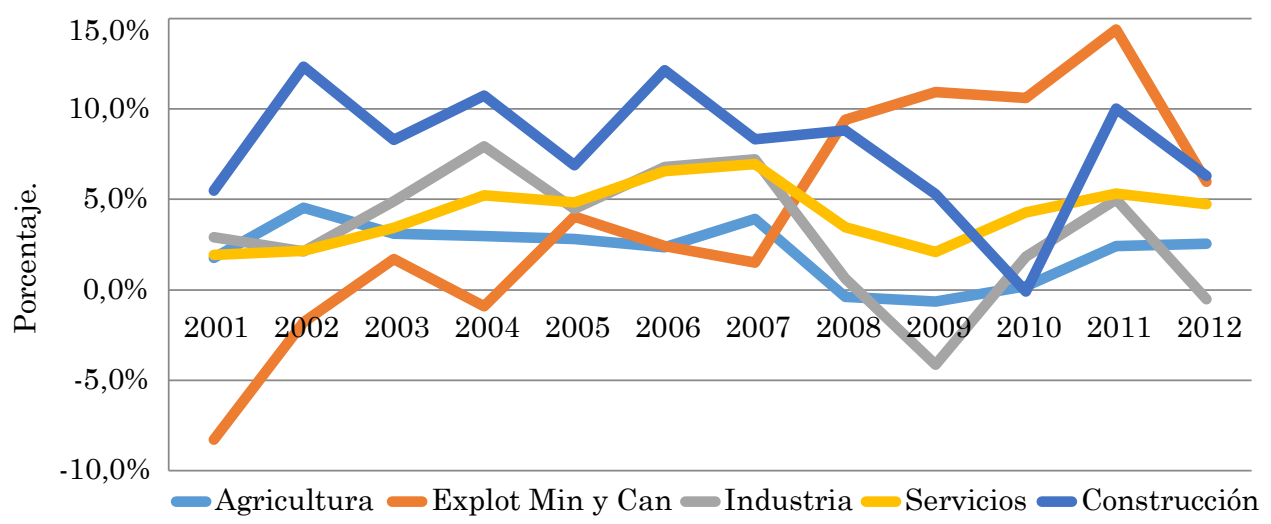

Gráfico 3. Crecimiento por ramas de actividad económica en la década del 2000.

Fuente: elaboración propia a partir del DANE (2005, 2007, 2009, 2011, 2013).

Para analizar a un poco más profundo el comportamiento del sector servicios se hace necesaria la desagregación del mismo, ob- servando el comportamiento de cada uno de los sub-sectores que lo componen, los cuales son: comercio; transporte, almacenamiento 
y comunicaciones; establecimientos financieros; servicios sociales y comunales; y suministro de electricidad, gas y agua.

Comportamientos de los subsectores que componen el sector de servicios. $\mathrm{Al}$ analizar el sector de servicios de forma general, y al hacer una comparación de su composición en los años 2000 y 2012, se encuentra que su estructura fue la misma; hubo un incremento en la participación en los subsectores de comercio y establecimientos financieros de un 1\% aproximadamente, mientras que los servicios sociales y comu- nales disminuyeron su participación pasando de un $29 \%$ en 2000 a un $26 \%$ en 2012 .

También se observa que el subsector que más aporto a PIB fue el de establecimientos financieros, seguros, actividades inmobiliarias y servicios a las empresas con un $33 \%$ aproximadamente, como se pone en evidencia en el gráfico 3, a éste le sigue el subsector de servicios sociales, personales y comunales con un $28 \%$ aproximadamente, mientras el subsector que aportó en menor medida al PIB fue el de suministro de electricidad, gas y agua con un $7 \%$ aproximadamente.
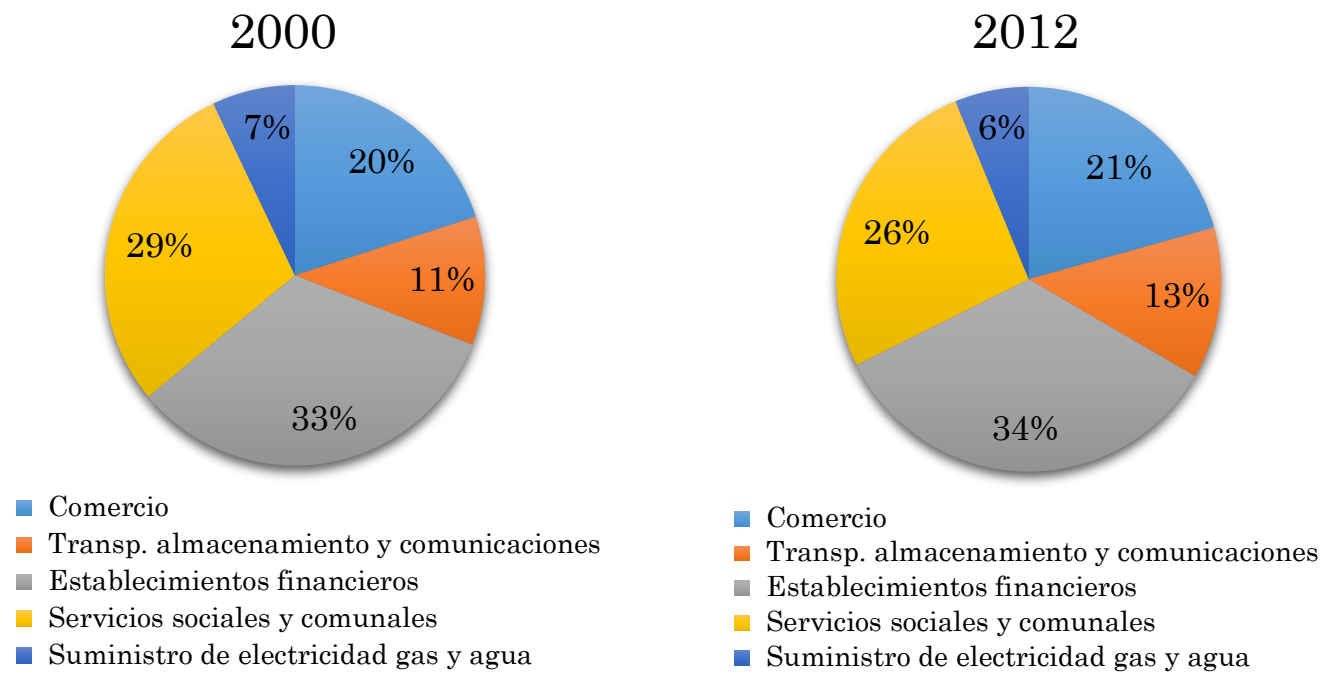

Gráfico 4. Composición del sector de servicios por subsectores en 2000 y 2012.

Fuente: elaboración propia a partir del DANE (2005, 2007, 2009, 2011, 2013). 
Al desagregar el sector de servicios y obtener la participación de los subsectores al PIB (gráfico 4), se encontró que el subsector de establecimientos financieros, seguros, actividades inmobiliarias y servicios a las empresas contribuyen al PIB en un $19,2 \%$ en promedio, siendo éste el subsector con mayor aporte dentro del sector de servicios, además de tener en general un comportamiento uniforme en la década.

En seguida se encuentra el subsector asociado con servicios sociales, comunales y personales con una participación del 15,83\% en promedio a lo largo de la década, siendo el segundo con mayor participación de los subsectores del sector terciario. El comercio aparece ubicado en tercer lugar con un aporte significativo al PIB de $12 \%$ en promedio, como se pone en evidencia en la tabla 3; esta cifra refleja la importancia de este subsector, no sólo en el sector de servicios sino, además, en toda la economía, debido a que permite la interrelación en los sectores productivos.

Con respecto al subsector de transporte, almacenamiento y comunicaciones se puede decir que el desarrollo y sostenimiento de una economía dependen en gran medida de la capacidad de transportar, almacenar y tener una excelente infraestructura vial, sin dejar atrás la importancia de las comunicaciones como herramienta fundamental para el desarrollo; el aporte de este subsector al PIB fue en promedio del $7 \%$ en la década.

Vale la pena mencionar que en Colombia la red vial se ha mantenido rezagada, convirtiéndose en una de las razones por la que en los planes de desarrollo se venga incluyendo la necesidad de mejorar y construir redes de carreteras, aeropuertos, puertos y vías fluviales que contribuyan al desarrollo de la economía por medio de la conexión de las diferentes regiones del país y, así, se contribuya a mejorar los aspectos relacionados con el comercio exterior.

\section{La innovación y su financiación en el sector de servicios en Colombia}

A continuación se desagrega la innovación presentada en el sector de servicios basados en los datos suministrados en la Encuesta de Desarrollo e Innovación Tecnológica en el Sector de Servicios (EDITS), que permiten analizar de forma detallada el comportamiento de la innovación y el personal ocupado en ese sector, además de la relación entre estas variables para determinar la incidencia del empleo en los procesos innovativos del sector de servicios.

Analizando la innovación en el periodo estudiado se encontró que la innovación de proceso es la que más aporta a las actividades de innovación con un $46 \%$ en el total de 
las innovaciones presentadas en el periodo estudiado, registrando su mayor aporte en el periodo $2008-2009$ con un $49 \%$ de las innovaciones presentadas. En segundo lugar se encuentra la innovación de producto con un $28 \%$ en las innovaciones totales, teniendo su mayor aporte en el periodo 2008-2009 con un 59\% de participación.

En general, la innovación del sector de servicios presenta un crecimiento del periodo 2004-2005 al 2008-2009 de un 27\%, incrementando en 12.378 las innovaciones totales, donde se percibe un cambio en la estructura de participación de los tipos de innovación.
En 2004-2005, la innovación organizativa representaba el $56 \%$ del total de innovaciones mientras que en 2008-2009 pasó a un tercer lugar con un $23 \%$, donde la innovación de proceso ocupó el primer lugar con un $48 \%$ de la participación. Ya para el periodo 2008-2009 a 2010-2011, se nota una disminución de 2.352 innovaciones equivalentes a un $13 \%$ de las innovaciones totales, donde la innovación de proceso continúa posicionándose en el primer lugar con un 51\% de participación, indicando que la mayoría de la innovaciones presentadas del sector de servicios obedecen a cambios generados al interior de las estructuras productivas don-

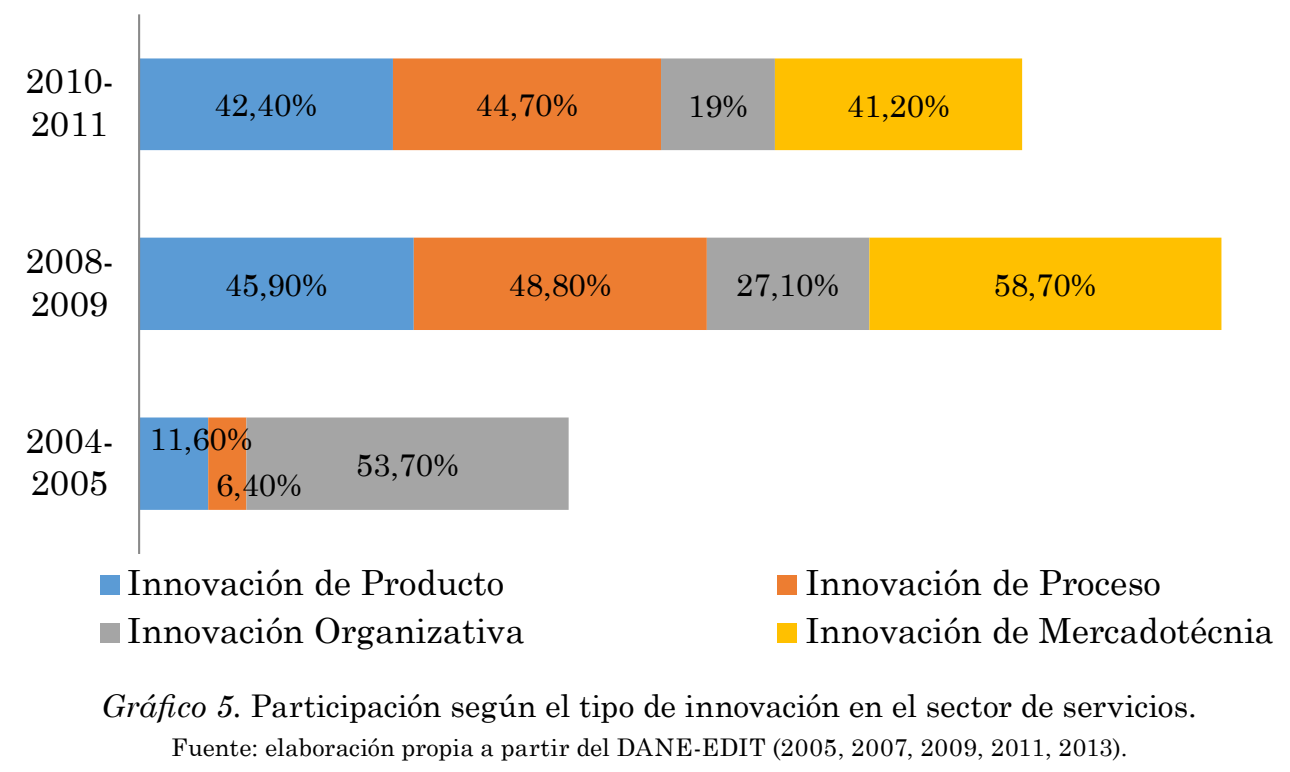


de se mejoraron o se crearon nuevas técnicas de producción.

Financiamiento de las actividades de desarrollo e innovación tecnológica. Las empresas tienen diversas opciones para financiar sus proyectos y programas de desarrollo tecnológico. La primera opción son las líneas, proyectos y programas que provee el Estado colombiano como parte de la política de apoyo empresarial. La segunda opción son los recursos que provee el sector privado a través de la banca privada, el sector externo y las mismas empresas.

2008

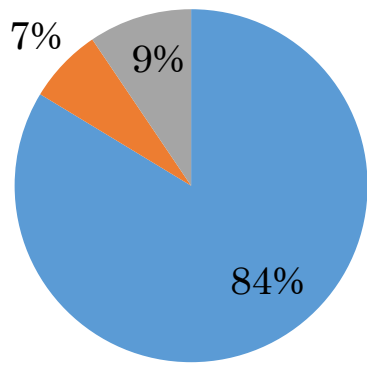

- Recursos propios de la empresa 84\% Año 2008

- Recursos publicos 7\% Año 2008

- Recursos de banca privada y otros a 9\% Año 2008
El valor financiado para actividades de desarrollo e innovación tecnológica durante 2008 llegó a $\$ 124.978 .505$ billones, mientras que para el año 2011, sumó $\$ 122.348 .937$ billones distribuidos así: las fuentes del sector público (cofinanciación y acceso a crédito) participan para el año 2008 con el 7\% (\$68.263.002 billones) y para el 2011 con el 4\% (\$98.563.524 billones); la banca privada participa con el $9 \%$ y $27 \%$ para los dos años, respectivamente; en los recursos propios de la empresa se puede observar que se tiene la mayor participación con un $84 \%$ para el año 2008 y $69 \%$ para el 2011 .

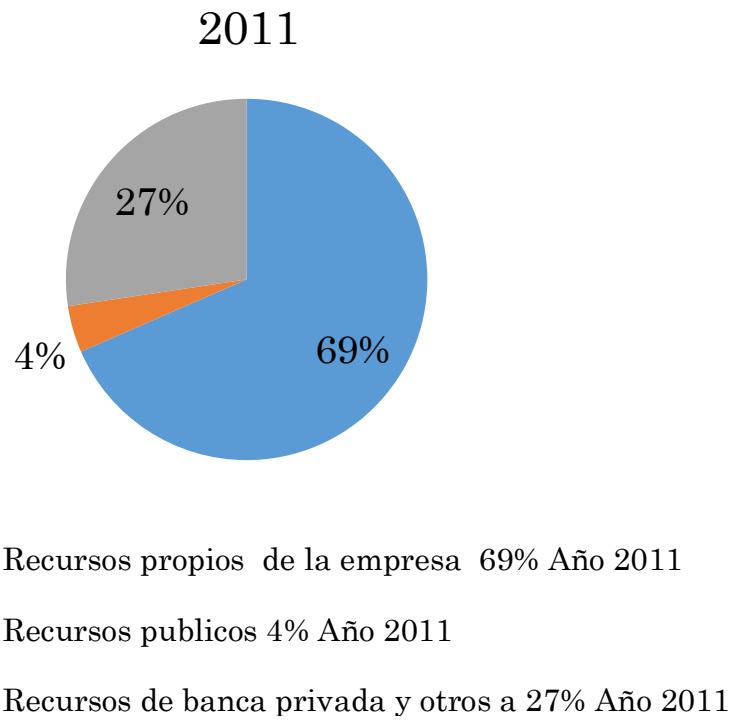

Gráfico 6. Participación de la inversión en ACTI por grupos de financiación.

Fuente: elaboración propia a partir del DANE-EDIT $(2009,2011,2013)$. 
Financiamiento con recursos públicos de las ACTI. Según las cifras de la Encuesta de Desarrollo e Innovación Tecnológica, los empresarios tienen una relati-

2008

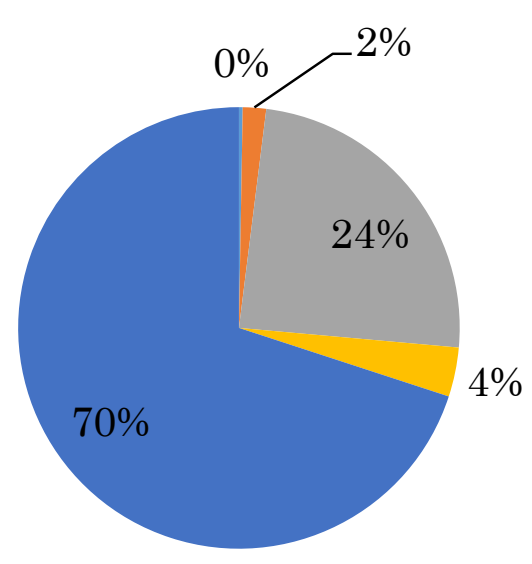

- COFINANCIACION 2008 FOMIPYME (Línea de innovación, desarrollo y transferencia tecnológica) $0 \%$

- COFINANCIACION 2008 SENA

(Programa Innovación y Desarrollo Tecnológico Ley 344/96) $2 \%$

- COFINANCIACION 2008 COLCIENCIAS Universidad CIA-CDT-Empresa 24\% va participación de las fuentes de cofinanciación del sector público. Los programas del SENA, según la Ley 344 de 1996 (faculta a invertir el $2 \%$ de sus recursos en

Gráfico 7. Línea de cofinanciación del sector público.

Fuente: elaboración propia a partir del DANE-EDIT $(2009,2011,2013)$.

- COFINANCIACION 2011 FOMIPYME (Línea de innovación, desarrollo y transferencia tecnológica) $1 \%$

- COFINANCIACION 2011 SENA (Programa Innovación y Desarrollo Tecnológico Ley 344/96) 1\%

- COFINANCIACION 2011 COLCIENCIAS Universidad CIA-CDT-Empresa 25\% 
programas y proyectos de competitividad y desarrollo tecnológico productivo), en el 2008 y en el 2011 tienen un porcentaje de sus recursos en los proyectos de competitividad y desarrollo tecnológico productivo del $1 \%$, teniendo una gran reducción de capital enfocado a estos proyectos. También, Colciencias Universidad CIA-CDT Empresa tuvo en el año 2008, el 24\% de las investigaciones en actividades científicas, tecnológicas y de innovación, y en el 2011, el $25 \%$.
Ya que Colciencias-universidad tiene una participación del $25 \%$, en Colciencias el riesgo tecnológico compartido fue del 4\% y $7 \%$ para cada año. En el Ministerio de Agricultura y Desarrollo Rural se obtienen los más altos porcentajes en el 2008: la participación en los programas de investigación fue del 70\%, y en el 2011 se presentó una reducción, pero el Ministerio de Agricultura y Desarrollo Rural obtuvo una participación del $66 \%$ en estos proyectos de investigación.

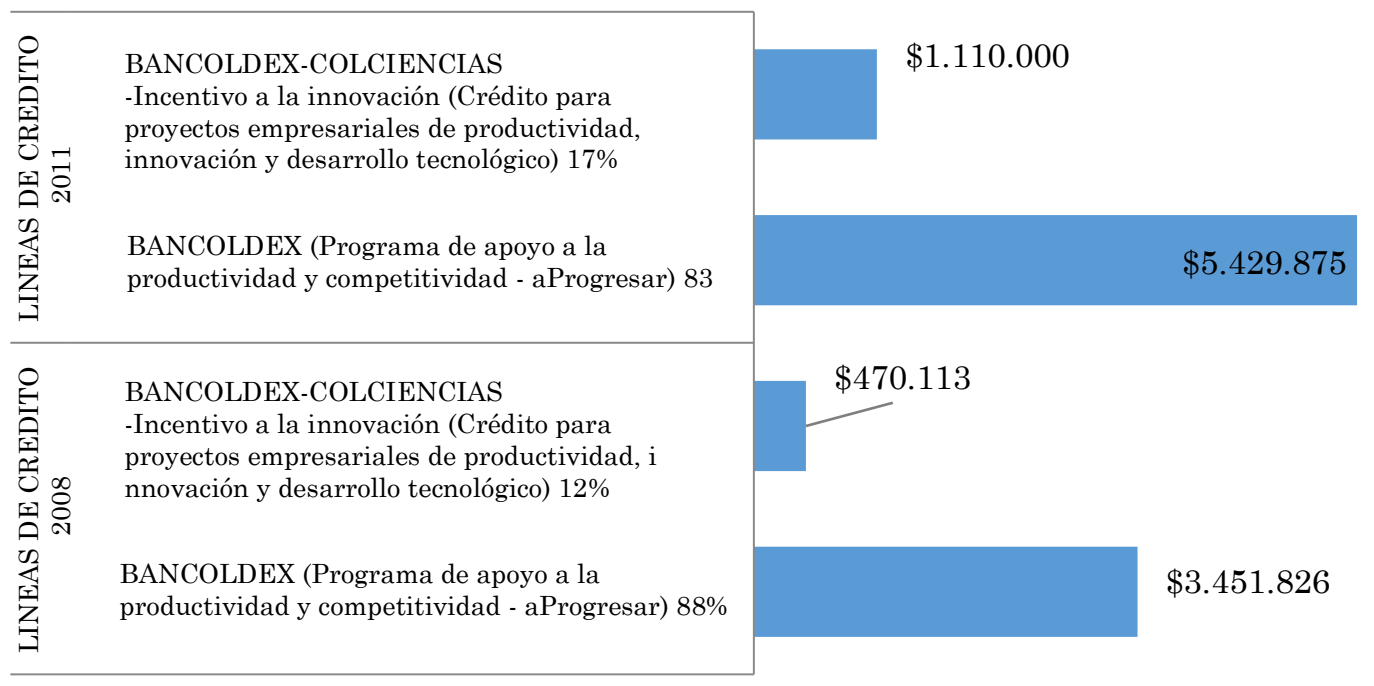

Gráfico 8. Línea de cofinanciación del sector público y división económica.

Fuente: elaboración propia a partir del DANE-EDIT (2005, 2007, 2009, 2011, 2013). 
2008

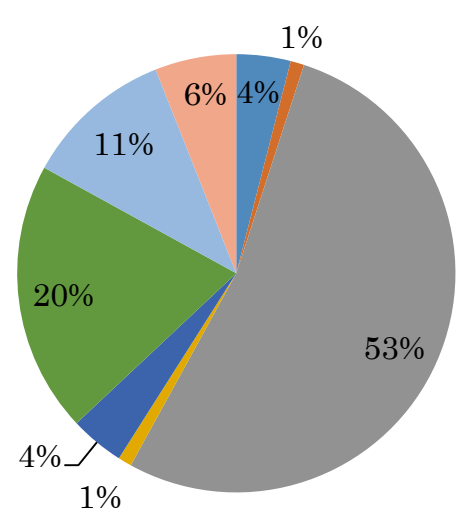

- Suministro de electricidad, gas, vapor y agua caliente

- Captación, depuración y distribución de agua

- Centros de investigación y desarrollo

- Transporte de carga por carretera

- Telecomunicaciones

- Actividades relacionadas con la salud humana

- Actividades de cinematografía, radio y televisión y otras actividades de entretenimiento

- Educación superior

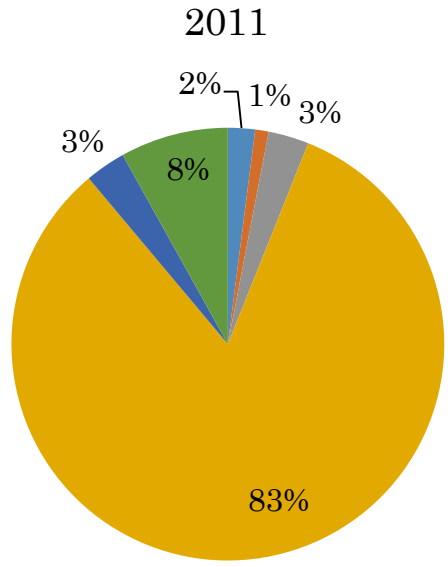

- Captación, depuración y distribución de agua

- Comercio al por mayor, excepto el comercio de vehículos automotores

- Transporte por vía aérea

- Centros de investigación y desarrollo

- Actividades relacionadas con la salud humana

- Educación superior privada

Gráfico 9. Financiación del sector público por servicios más representativos. Fuente: elaboración propia a partir del DANE-EDIT (2009, 2011, 2013). 
El sector público, además, ofrece programas de acceso a crédito para actividades de desarrollo tecnológico. La fuente de financiación más conocida de este grupo es la modalidad de crédito BANCOLDEX que financia proyectos de innovación, desarrollo tecnológico y productividad a empresas exportadoras; el 88\% en el 2008 y, en el 2011, con el 83\%, las empresas acuden más a esta línea de crédito.

Los sectores escogidos son los más representativos, se presentan las variaciones del 2008 al 2011 por sectores. El sector que tiene el monto más alto por parte de la línea de cofinanciación del sector público. Para el 2011, éste corresponde a centros de investigación y desarrollo con un $82 \%$, equivalente a un monto de $\$ 102.187 .912$; para el año 2008, los centros de investigación y desarrollo tienen una participación del 53\% con un monto de $\$ 64.126 .406$.

La línea de cofinanciación por parte del sector público del 2008 al 2011 aumentó en un $29 \%$, esto corresponde a un monto de $\$ 38.061 .506$; el servicio que obtuvo la segunda participación más alta fue la educación superior, que en el 2008 representaba un $6 \%$ y para el 2011, un 8\%. En estos gráficos se puede observar que la actividades relacionadas con la salud humana han tenido una reducción importante del 2008 al 2011, éstas tuvieron un descenso del 17\%, esto equivale a $\$ 20.910 .767$; los otros servicios analizados tienen una participación muy baja.

En estos gráficos se analiza la participación de los servicios más representativos. Se empezará con las actividades relacionadas con la salud humana, la cual, en el 2008, obtuvo el $41 \%$ con una cuantía de $\$ 68.116 .662$ y, para el 2011, estas actividades obtuvieron una intervención del $11 \%$ y una proporción de $\$ 68.116 .662$; se puede afirmar entonces que tanto la financiación por parte del sector público y la banca privada han dejado de financiar estos sectores.

Con respecto al comercio al por menor, excepto el comercio de vehículos automotores, en el 2011 tuvo una intervención del 47\% y en el 2008 sólo fue del 4\%, esto quiere decir que este sector es muy representativo para los interés de los empresarios, ya que creció un $43 \%$ en 4 años, pasando de $\$ 6.083 .714$ en el 2008 a $\$ 385.970 .325$ en el 2011. El comercio al por mayor, excepto el comercio de vehículos automotores, en el año 2008 obtuvo un $8 \%$ y en el 2011 un 7\%, así, se puede plantear que se mantuvo equilibrada. La participación del resto de sectores de la CIIU.Rev.3.A.C. son muy bajos, por esto hay sectores que no están en las gráficas ya que a la hora de la selección de sectores su participación era del 1\% o 0\%. 


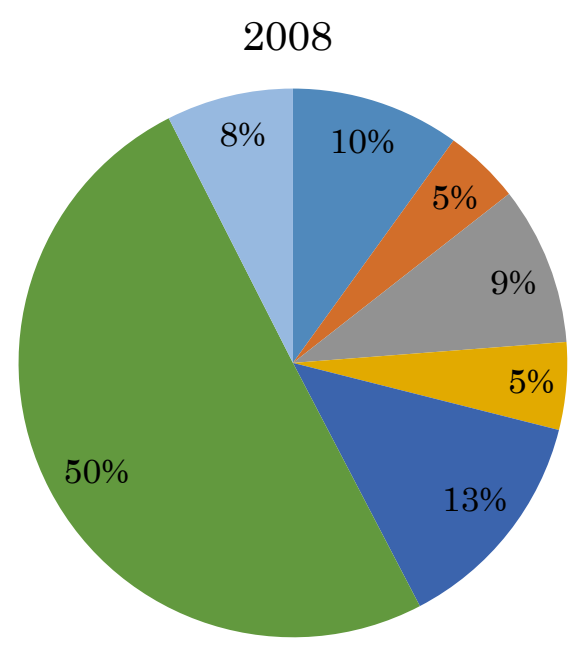

- Comercio al por mayor, excepto el comercio de vehículos automotores

- Comercio al por menor, excepto el comercio de vehículos automotores

- Informática y actividades conexas

- Transporte colectivo regular de pasajeros por via terrestre

- Transporte de carga por carretera

- Actividades relacionadas con la salud humana

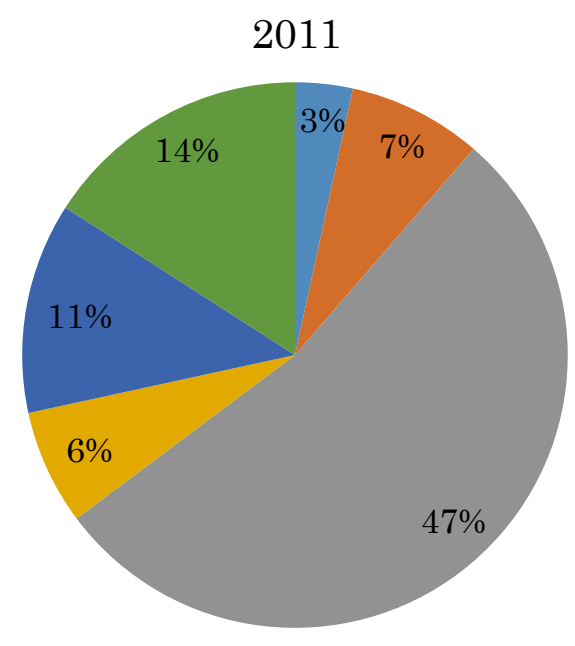

- Suministro de electricidad, gas, vapor y agua caliente

- Comercio al por mayor, excepto el comercio de vehículos automotores

- Comercio al por menor, excepto el comercio de vehículos automotores

- Centros de investigación y desarrollo

- Actividades relacionadas con la salud humana

- Correos y Telecomunicaciones

- Actividades de cinematografía, radio y televisión y otras actividades de entretenimiento

Gráfico 10. Financiación por parte de la banca privada.

Fuente: elaboración propia a partir del DANE-EDIT (2009, 2011, 2013). 

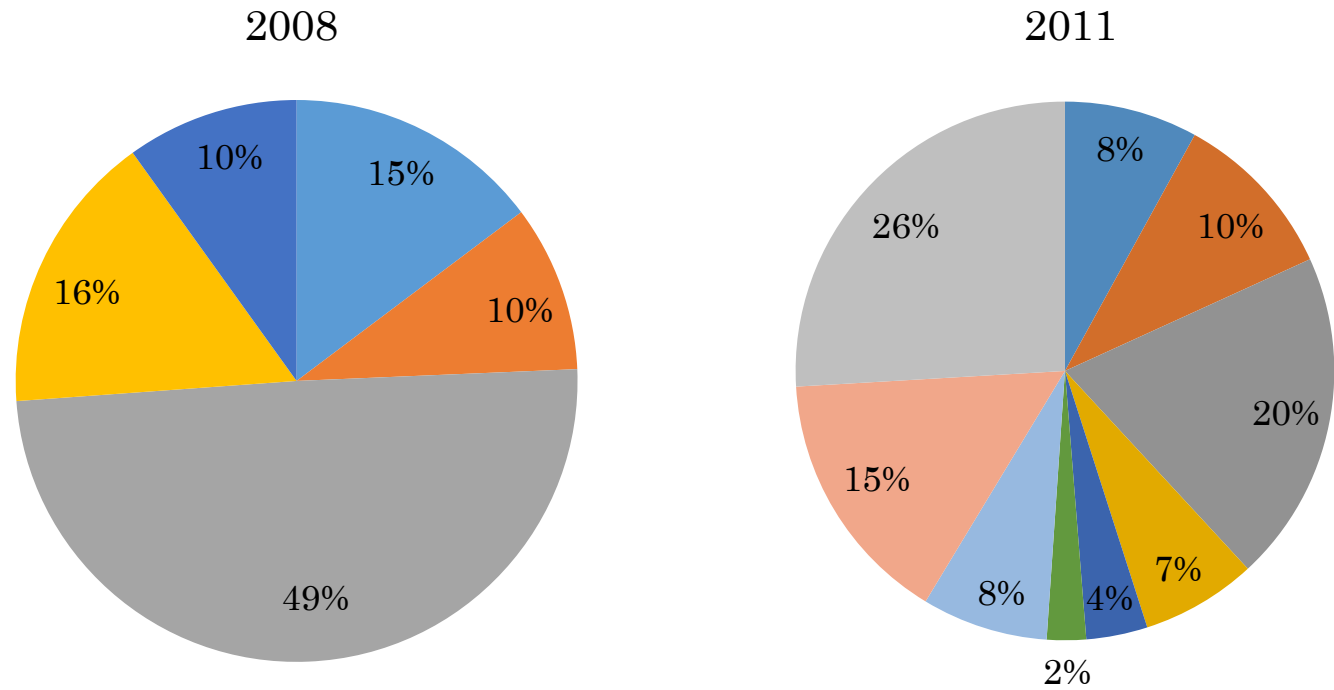

- Comercio al por mayor y en comisión o por contrata, excepto el comercio de vehículos automotores y motocicletas; mantenimiento y reparación de maquinaria y equipo

- Comercio al por menor, excepto el comercio de vehículos automotores y motocicletas; reparación de efectos personales y enseres domésticos

- Centros de investigación y desarrollo

Eliminación de desperdicios y aguas residuales, saneamiento y actividades similares

- Actividades de cinematografía, radio y televisión $\mathrm{y}$ otras actividades de entretenimiento

- Suministro de electricidad, gas, vapor y agua caliente

- Comercio al por mayor, excepto el comercio de vehículos automotores

- Comercio al por menor, excepto el comercio de vehículos automotores

- Informática y actividades conexas

- Centros de investigación y desarrollo

- Eliminación de desperdicios y aguas residuales, saneamiento y actividades similares

- Educación superior privada

- Correos y Telecomunicaciones

- Actividades bancarias

Gráfico 11. Financiación por parte de recursos propios.

Fuente: elaboración propia a partir del DANE-EDIT (2009, 2011, 2013). 
2008

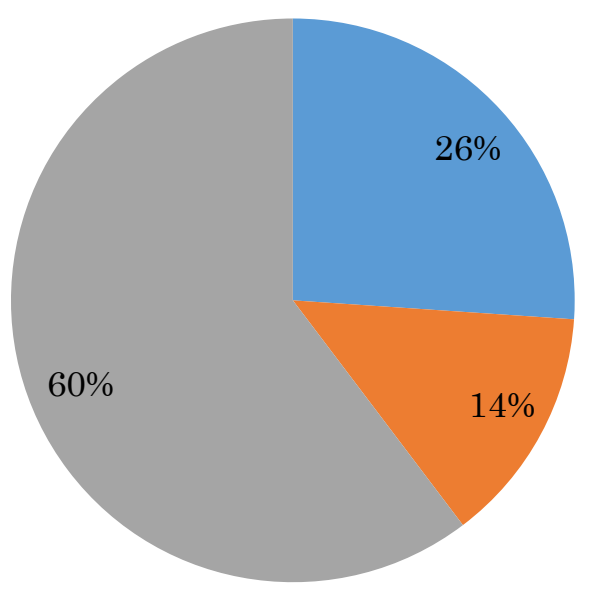

- Transporte colectivo regular de pasajeros por via terrestre

Actividades relacionadas con la salud humana

Actividades de cinematografía, radio y televisión y otras actividades de entretenimiento
2011

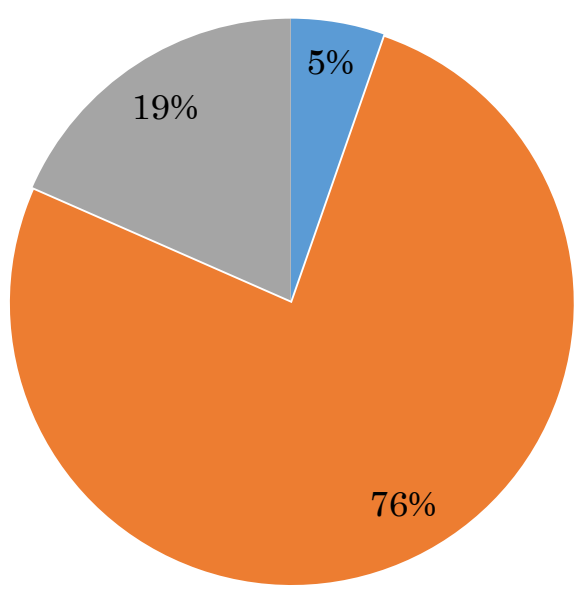

- Comercio de vehículos automotores, combustibles y lubricantes

- Transporte por vía aérea

- Actividades relacionadas con la salud humana

Gráfico 12. Financiamiento con la línea de crédito BANCOLDEX.

Fuente: elaboración propia a partir del DANE-EDIT (2009, 2011, 2013). 
La financiación por parte de los recursos propios de las empresas ha tenido unas grandes variaciones, por ejemplo, el sector de actividades bancarias, en el 2008, fue de un $1 \%$ y en el 2011 tiene una participación del $22 \%$, siendo el sector con mayor participación.

El comercio al por menor presentó un crecimiento importante pasando del $6 \%$ en el 2008 al $17 \%$ en el 2011. El comercio al por mayor obtuvo un descenso pasando del 10\% al 7\%. Así mismo, las gráficas permiten analizar el gran descenso que tuvo la financiación de centros de investigación y desarrollo por parte de los recursos de las empresas, pasando de $32 \%$ en el 2008 al $3 \%$ en el 2011; este descenso se debe a que el sector privado y público han invertido más en este sector. También se presenta un descenso en la eliminación de desperdicios y aguas residuales, pasando del 11\% en el 2008 al $2 \%$ en el 2011.

El financiamiento con recursos públicos de las actividades científicas, tecnológicas y de innovación para las empresas del sector de servicios son investigadas, según la línea de crédito BANCOLDEX, demostrando que la financiación es muy escasa porque sólo tres sectores son los que representan actividad. El sector de comercio de vehículos automotores, combustibles y lubricantes representa el 5\%, el transporte por vía aérea el $76 \%$, y las actividades relacionadas con la salud humana el 19\%, esto para el año 2011.

Para el año 2008, el transporte colectivo regular de pasajeros por vía terrestre tiene una participación del $26 \%$ y las actividades de cinematografía, radio y televisión y otras actividades de entretenimiento el $60 \%$. El único sector que en ambos periodos tiene participación es el de las actividades relacionadas con la salud humana, que en el año 2008 tuvo un 14\%, entonces se puede decir que este sector ha crecido un $5 \%$.

\section{Conclusiones}

El sector de servicios en Colombia ha venido ganando importancia en la economía debido a su creciente participación en el Producto Interno Bruto. En la década del 2000, alcanzó el 65\% y la cantidad de personal ocupado en $51 \%$. Con respecto a los tipos de innovación, la más representativa fue la innovación de proceso con un $46 \%$, seguida de la innovación de producto con un $28 \%$ y, en último lugar, se encuentra la innovación organizativa con un $26 \%$ (incluyendo la de mercadotecnia). 
La fuente de financiación más importante en actividades de desarrollo e innovación tecnológica en el periodo de estudio corresponde a los recursos de propios de las empresas, que en el 2008 presentó el $84 \%$, seguido de los recursos de la banca privada, y, por último, el sector público con una participación del 7\%. En el 2011, los recursos propios de las empresas disminuyen a un $69 \%$ pero sigue siendo en sector que más financia al sector de servicios y la innovación en Colombia.

Es importante resaltar que las líneas de financiación del sector público se concentran en el Ministerio de Agricultura y Desarrollo Rural en los programas de investigación, desarrollo tecnológico e innovación por cadenas productivas, ya que en el año 2008 fue del $70 \%$ y, en el 2011, del 66\%.

En la línea de crédito se pone en evidencia que BANCOLDEX tiene una financiación del 88\% con un monto de \$ 3.451 .826 en el 2008 y, en el 2011, del 83\% equivalente a un monto de $\$ 5.429 .875$, bajo el porcentaje pero el monto fue superior al del 2008.

En actividades científicas, tecnológicas y de innovación, los sectores más repre- sentativos, tanto en lo público, privado y recursos propios de las empresas, se observó que en el sector público los centros de investigación y desarrollo son los más financiados tanto para el 2008 como para el 2011; en la banca privada, el sector de actividades relacionadas con la salud humana tiene la financiación más alta (41\%) para el año 2008, y para el 2011 lo tiene el comercio al por menor con el 47\%; y con respecto a los recursos propios de las empresas, el sector de centros de investigación y desarrollo tiene una financiación del $32 \%$, siendo la más financiada en el periodo del 2008, sin embargo, en el 2011, el sector de actividades bancarias es el más representativo con un $22 \%$. De esta forma, se identificó que los sectores de investigación y desarrollo son los más financiados en el país.

\section{Referencias}

DANE. (2005). Encuesta de Desarrollo e Innovación Tecnológica en el Sector Servicios - EDIT - II 2003 - 2004. Recuperado de https://www.dane. gov.co/files/investigaciones/industria/innovacion_tecnol_ind_manufacturera.pdf 
DANE. (2007). Encuesta de Desarrollo e Innovación Tecnológica en el Sector Servicios - EDIT - III 2005 - 2006. Recuperado de https://www.dane.gov. co/files/investigaciones/boletines/edit/ boletin_EDIT_Manufacturera.pdf

DANE. (2009). Encuesta de Desarrollo e Innovación Tecnológica en el Sector Servicios - EDIT - IV 2007 - 2008. Recuperado de https://formularios. dane.gov.co/Anda_4_1/index.php/catalog/rdf/19

DANE. (2011). Encuesta de Desarrollo e Innovación Tecnológica en el Sector Servicios - EDIT - V 2009 - 2010. Recuperado de https://formularios. dane.gov.co/Anda_4_1/index.php/catalog/rdf/218

DANE. (2013). Encuesta de Desarrollo e Innovación Tecnológica en el Sector Servicios - EDIT - VI 2011 - 2012. Recuperado de https://www.dane. gov.co/files/investigaciones/boletines/edit/boletin_EDIT_Manufacturera_2011_2012.pdf

Freeman, C. (1994). La innovación y el crecimiento. En Dogson M. y Rothwell (ed.), El manual de la innovacion industrial. Reino Unido: Elgar, Edward Publishing.
Freeman, C., Clark, J. y Soete, L. (1985). Desempleo e innovación tecnológica. Madrid: Ministerio de Trabajo y Seguridad Social.

Freeman, C. (1988). Política de tecnología y desempeño económico: Lecciones de Japón. Londres: Pinter.

ISEA (2008). Análisis prospectivo sobre Modelos de Innovación en el sector servicios. Ministerio de Industria, Turismo y Comercio, del Programa de Centros del Conocimiento y Contenidos Digitales, en el marco del Plan Avanza. [Informe]. Recuperado de http://www.iseamcc.net/eISEA/ Vigilancia_tecnologica/informe_1. pdf

Porter, M. (1990). The competitive advantage of notions. Harvard business review, 68(2), 73-93.

Ruttan, V. (1979). Usher y Schumpeter en la invención, la innovación y el cambio tecnológico. En N. Rosenberg, Economía del cambio tecnológico. México: Fondo de Cultura Económica.

Schumpeter, J. (1971). Capitalismo, socialismo y democracia. Madrid: Aguilar. 
Schumpeter, J. (1979). La teoría del desenvolvimiento económico. México D.F: Fondo de Cultura Económica.
UNED. (2010). Resumen del manual de Oslo sobre innovación. Obtenido de http://portal.uned.es/portal/page?_pageid $=93,23280929 \& \_d a d=$ portal 